\title{
High energy QCD Lipatov's effective action in Euclidean space
}

\author{
S. Bondarenko ${ }^{\mathrm{a}}$ \\ Physics Department, Ariel University, Ariel 40700, Israel
}

Received: 5 February 2020 / Accepted: 14 April 2020 / Published online: 4 May 2020

(C) The Author(s) 2020

\begin{abstract}
The continuation of high energy QCD Lipatov's effective action to Euclidean space is performed. The resulting Euclidean QCD RFT action is considered separately in Euclidean "light-cone" coordinates and axial gauge suitable for the numerical and analytical calculations correspondingly. The further application of the obtained results is also discussed.
\end{abstract}

\section{Introduction}

Lipatov's effective action approach, [1-13], can be considered as Regge Field Theory (RFT) constructed on the base of QCD and intended to to take account of unitarity corrections to the high-energy scattering amplitudes in the multi-Regge kinematics, see [14-35]. It also can be considered as a further generalization of phenomenological RFT [36-52]. The formalism is based on the reggeized gluons (reggeons) as the main degrees of freedom, with the Pomeron calculus, [5368], introduced on the base of colorless Reggeons states.

The transition to QCD RFT formulation of the theory is performed with the introduction of the generating functional for the reggeized gluons fields $A_{ \pm}$obtained by an integration out of the gluon fields $v$ from the the $S_{e f f}[v, A]$ :

$e^{\imath \Gamma[A]}=\int D v e^{\imath S_{e f f}[v, A]}$

with Lipatov's effective action defined as QCD action plus effective currents for the longitudinal gluon fields:

$$
\begin{aligned}
S_{e f f}= & -\int d^{4} x\left(\frac{1}{4} G_{\mu \nu}^{a} G_{a}^{\mu \nu}+\operatorname{tr}\left[\left(\mathcal{T}_{+}\left(v_{+}\right)\right.\right.\right. \\
& \left.\left.\left.-A_{+}\right) j_{r e g}^{+}+\left(\mathcal{T}_{-}\left(v_{-}\right)-A_{-}\right) j_{r e g}^{-}\right]\right)
\end{aligned}
$$

\footnotetext{
a e-mail: sergeyb@ariel.ac.il (corresponding author)
}

and effective currents defined as

$\mathcal{T}_{ \pm}\left(v_{ \pm}\right)=\frac{1}{g} \partial_{ \pm} O\left(v_{ \pm}\right)=v_{ \pm} O\left(v_{ \pm}\right)$,

$j_{\text {reg } a}^{ \pm}=\frac{1}{C(R)} \partial_{i}^{2} A_{a}^{ \pm}$

where $C(R)$ is eigenvalue of Casimir operator in the representation $\mathrm{R}, \operatorname{tr}\left(T^{a} T^{b}\right)=C(R) \delta^{a b} A_{ \pm}$as Reggeon fields, see [1-9]. This action can be considered as an averaging of the interacting eikonal lines over the gluon fields, this definition and the derivation of the Lipatov's effective action were clarified in [11] paper, see also [12]. In general, the form of the Lipatov's operator $O$ (and correspondingly $\mathcal{T}$ ) depends on the particular process of interests, see again in [11], the simplest choice of the operators are Wilson lines (ordered exponential) of the longitudinal gluon fields:

$O\left(v_{ \pm}\right)=P e^{g \int_{-\infty}^{x^{ \pm}} d x^{ \pm} v_{ \pm}\left(x^{+}, x^{-}, x_{\perp}\right)}, \quad V_{ \pm}=\imath T^{a} v_{ \pm}^{a}$,

see also [28-35]. There are additional kinematical constraints for the Reggeon fields

$\partial_{-} A_{+}=\partial_{+} A_{-}=0$,

corresponding to the strong-ordering of the Sudakov components in the multi-Regge kinematics, see [1-7,11]. The action is constructed by the request that the $\mathrm{LO}$ value of the classical gluon fields in the solutions of equations of motion will be fixed as

$v_{ \pm}^{c l}=A_{ \pm}$

this condition also can be considered as the definition of the Reggeon fields. The further use of the Eq. (2) action is based on the different perturbative calculation schemes, see [11-35], of course all the calculations are performed in the Minkowski space. 
The continuation of the action to the Euclidean space, respectively, is an important task due to the two interesting issues. The fist one is the formulation of the action for the possible lattice (numerical) calculation of any objects of interests in the Euclidean space. The second one is the analytical investigation of the non-perturbative contributions, such as instantons for example, to the Reggeons interactions vertices and amplitudes. There are many calculations which have be done in this direction, see for example [69-86], but the Lipatov's action continued to Euclidean space can serve as some generic approach for the consistent account of the instanton-Reggeon interactions and instanton contributions to the high-energy scattering amplitudes. Therefore, the paper is organized as follows. In the next section we introduce all required tools and notions for the continuation of the action to Euclidean space. In the Sect. 3 we consider the continuation of the Lipatov's action in the Euclidean space. Section 4 and 5 are about the different forms of the action. In Sect. 4 we discuss the action written in the terms of new coordinates similar to the light-cone coordinates of Minkowski space, we will call it further as Euclidean "light-cone" coordinates. In the Sect. 5 we consider the axial gauge for the action which is suitable for the analytical calculations in the framework. The last section is Conclusion where the further applications of the results are discussed.

\section{Euclidean space kinematics}

The main task of the problem is the continuation of the effective currents of the action to Euclidean space. We begin from the usual four-momentum vectors in Minkowski space used for the description of the high energy scattering kinematics:

$p_{1}^{\mu}=\frac{\sqrt{s}}{2}\left(1,1,0_{\perp}\right), \quad p_{2}^{\mu}=\frac{\sqrt{s}}{2}\left(1,-1,0_{\perp}\right)$,

where

$s=\left(p_{1}+p_{2}\right)^{2}, \quad t=\left(p_{1}-p_{1}^{\prime}\right)^{2}, \quad s=\left(p_{1}-p_{2}^{\prime}\right)^{2}$.

In the light cone coordinate frame these vectors can be rewritten as

$p_{1 L . C .}^{\mu}=\sqrt{\frac{s}{2}}\left(1, \beta, 0_{\perp}\right), \quad p_{2 L . C .}^{\mu}=\sqrt{\frac{s}{2}}\left(\beta, 1,0_{\perp}\right)$

with $\beta$ as some regularization introduced in order to regularize the rapidity divergences of the corresponding integrals, see [87-92] for example. Application of these vectors to the Eq. (3) current terms provides

$\sqrt{\frac{2}{s}} p_{1 L . C}^{\mu} \partial_{\mu} \stackrel{\beta \rightarrow 0}{\rightarrow} \partial_{+}, \quad \sqrt{\frac{2}{s}} p_{2 L . C .}^{\mu} \partial_{\mu} \stackrel{\beta \rightarrow 0}{\rightarrow} \partial_{-}$ as requested. Therefore, the Eikonal interaction term introduced in [11,12], see also Eq. (29) below, can be written as following:

$\frac{2}{s}\left(p_{1 L . C .}^{\mu} \partial_{\mu} O\left(v_{+}\right)\right) \partial_{\perp}^{2}\left(p_{2 L . C .}^{v} \partial_{v} O\left(v_{-}\right)\right)$

which is fully covariant, i.e. it can be also in the usual Minkowski coordinates as

$\frac{2}{s}\left(p_{1}^{\mu} \partial_{\mu} O\left(v_{+}\right)\right) \partial_{\perp}^{2}\left(p_{2}^{v} \partial_{\nu} O\left(v_{-}\right)\right)$,

that can considered as a step to the continuation of the effective action to Euclidean space. Nevertheless, it is not easy to work with $\beta$ as parameter performing the continuation for the arbitrary angle between particles trajectories in the Euclidean space, see [93,94]. Therefore, considering the hyperbolic angle between the light cone directions in the Minkowski space, we introduce the following vectors of the directions of the relativistic particles motion in the Minkowski space:

$n_{1}^{\mu}=\frac{1}{\sqrt{2}}\left(1, \tanh (\gamma / 2), 0_{\perp}\right)$

$n_{2}^{\mu}=\frac{1}{\sqrt{2}}\left(1,-\tanh (\gamma / 2), 0_{\perp}\right)$,

with the following form of the same vectors in the light cone coordinates:

$n_{+ \text {L.C. }}^{\mu}=\frac{1}{2}\left(1+\tanh (\gamma / 2), 1-\tanh (\gamma / 2), 0_{\perp}\right)$,

$n_{-L . C .}^{\mu}=\frac{1}{2}\left(1-\tanh (\gamma / 2), 1+\tanh (\gamma / 2), 0_{\perp}\right)$.

As usual, for the

$p^{2}=m^{2}$

we have at high energy

$\gamma \approx \ln \left(s / m^{2}\right), \quad \beta=m^{2} / s$

The Wick rotation of the vectors to the Euclidean space can be done now by

$\gamma \rightarrow 2 \imath \phi$

continuation, where $2 \phi$ is an angle between trajectories of the two particles in the c.m.f. in Euclidean space. We obtain correspondingly for Eq. (13):

$n_{1 E}^{\mu}=\frac{1}{\sqrt{2}}\left(1, \imath \tan (\phi), 0_{\perp}\right)$,

$n_{2 E}^{\mu}=\frac{1}{\sqrt{2}}\left(1,-l \tan (\phi), 0_{\perp}\right)$, 
the transforms

$\partial_{0} \rightarrow \imath \partial_{4 E}, \quad v_{0}^{a} \rightarrow \iota v_{0 E}^{a}$

must be performed further as well. Therefore we obtain

$n_{1}^{\mu} \partial_{\mu} \rightarrow \imath n_{+E}^{\mu} \partial_{\mu E}$,

$n_{2}^{\mu} \partial_{\mu} \rightarrow \imath n_{-E}^{\mu} \partial_{\mu E}$

where

$n_{+E}^{\mu}=\frac{1}{\sqrt{2}}\left(1, \tan (\phi), 0_{\perp}\right)$

$n_{-E}^{\mu}=\frac{1}{\sqrt{2}}\left(1,-\tan (\phi), 0_{\perp}\right)$

are vectors in the Euclidean space. Correspondingly, the Eikonal interaction term in Minkowski space can be rewritten in Euclidean space as

$$
\begin{aligned}
& -l \int d^{4} x\left(\left(n_{1}^{\mu} \partial_{\mu} O\left(v_{+}\right)\right) \partial_{\perp}^{2}\left(n_{2}^{\nu} \partial_{\nu} O\left(v_{-}\right)\right)\right) \\
& \rightarrow \int d^{4} x_{E}\left(\left(n_{+E}^{\mu} \partial_{\mu E} O_{E+}\right) \partial_{\perp E}^{2}\left(n_{-E}^{\mu} \partial_{\mu E} O_{E-}\right)\right) .
\end{aligned}
$$

with

$$
\begin{aligned}
& O\left(v_{ \pm}\right)=P e^{g \int_{-\infty}^{1} d \lambda\left(n_{1,2}^{\mu} v_{\mu}\right)} \\
& \rightarrow O_{E \pm}=P e^{\imath g \int_{-\infty}^{1} d \lambda\left(n_{ \pm E}^{\mu} v_{\mu E}\right)} \\
& v_{\mu E}=\imath T^{a} v_{\mu}^{a} .
\end{aligned}
$$

The remaining part of Eq. (2) gluon's QCD Lagrangian is continued to the Euclidean space as usual.

We note, that the exponential Eq. (23) is real in the case of the fundamental representation of the gluon field but remains imaginary for the adjoint representation of the field with $T_{b c}^{a}=-\imath f_{a b c}$. In this case we perform another continuation in the Euclidean space based on the following continuation of the coordinates and fields:

$$
\begin{aligned}
& \partial_{i} \rightarrow-l \partial_{i E}, \quad v_{i}^{a} \rightarrow-l v_{i E}^{a}, \\
& \partial_{0} \rightarrow \partial_{4 E}, \quad v_{0}^{a} \rightarrow v_{4 E}^{a} .
\end{aligned}
$$

This way of continuation still provides the correct continuation of the action into the Euclidean space:

$S=\int d^{3} x_{i} d t\left(\frac{1}{2} G_{0 i}^{a} G_{0 i}^{a}-\frac{1}{4}\left(G_{i j}^{a}\right)^{2}\right) \rightarrow \iota S_{E}$,

but with effective currents changed correspondingly to Eq. (24) transforms:

$$
n_{1}^{\mu} \partial_{\mu} \rightarrow n_{+E}^{\mu} \partial_{\mu E}, \quad n_{2}^{\mu} \partial_{\mu} \rightarrow n_{-E}^{\mu} \partial_{\mu E}
$$

with

$$
\begin{aligned}
& -l \int d^{4} x\left(\left(n_{1}^{\mu} \partial_{\mu} O\left(v_{+}\right)\right) \partial_{\perp}^{2}\left(n_{2}^{\nu} \partial_{\nu} O\left(v_{-}\right)\right)\right) \\
& \rightarrow \int d^{4} x_{E}\left(\left(n_{+E}^{\mu} \partial_{\mu E} O_{E+}\right) \partial_{\perp E}^{2}\left(n_{-E}^{\mu} \partial_{\mu E} O_{E-}\right)\right)
\end{aligned}
$$

and

$O_{E \pm}=P e^{g \int_{-\infty}^{1} d \lambda\left(n_{ \pm E}^{\mu} v_{\mu E}\right)}, \quad v_{\mu E}=f^{a} v_{\mu}^{a}$.

We see, that in this case the whole effective action remains real as well.

\section{Lipatov's action in Euclidean space}

In this Section we consider the generating functional for the Lipatov's operators in the Euclidean space, here and further we omit the $E$ notation in the formulae and discuss firstly the case of the adjoint representation of the gluon fields. ${ }^{1}$ We write the full Lagrangian of the approach as a continuation in the Euclidean space of the Eq. (2) Lagrangian of the interacting eikonal lines. Then the following expression is obtained:

$$
\begin{aligned}
Z[J]= & \frac{1}{Z^{\prime}} \int D v \exp \left(-S_{Y M}[v]+\frac{1}{2 g^{2} C(R)}\right. \\
& \times \int d^{4} x\left(n_{+}^{\mu} \partial_{\mu} O_{+}\right) \partial_{\perp}^{2}\left(n_{-}^{\mu} \partial_{\mu} O_{-}\right) \\
& -\frac{1}{2 g C(R)} \int d^{4} x J_{-}\left(n_{+}^{\mu} \partial_{\mu} O_{+}\right)-\frac{1}{2 g C(R)} \\
& \left.\times \int d^{4} x J_{+}\left(n_{-}^{\mu} \partial_{\mu} O_{-}\right)\right),
\end{aligned}
$$

the external currents $J_{ \pm}$here are the auxiliary ones, we take them equal to zero at the end of the derivation. Introducing Lipatov's operators

$\mathcal{T}_{ \pm}=\frac{1}{g} n_{ \pm}^{\mu} \partial_{\mu} O_{ \pm}=\left(n_{ \pm}^{\mu} V_{\mu}\right) O_{ \pm}$

we rewrite the same generating functional as

$$
\begin{aligned}
& Z[J] \\
& =\frac{1}{Z^{\prime}} \int D \operatorname{vexp}\left(-S_{Y M}[v]+\frac{1}{2 C(R)} \int d^{4} x \mathcal{T}_{+} \partial_{\perp}^{2} \mathcal{T}_{-}-\right. \\
& \left.\quad-\frac{1}{2 C(R)} \int d^{4} x J_{-} \mathcal{T}_{+}-\frac{1}{2 C(R)} \int d^{4} x J_{+} \mathcal{T}_{-}\right) .
\end{aligned}
$$

1 The case of the fundamental representation can be considered similarly to the adjoint one with $A_{ \pm}^{a} \rightarrow \iota A_{ \pm}^{a}$ redefinition of Reggeon fields which preserves the whole expression real, see end of the section. 
Now, with the help of some auxiliary fields ${ }^{2} A_{ \pm}$we obtain for the generating functional the following expression:

$$
\begin{aligned}
& Z[J] \\
& =\frac{1}{Z^{\prime}} \int D_{v} D A \exp \left(-S_{Y M}[v]-\frac{2}{C(R)}\right. \\
& \quad \times \int d^{4} x A_{+}(x) \partial_{\perp}^{2} A_{-}(x) \\
& \quad+\frac{1}{C(R)} \int d^{4} x \mathcal{T}_{+} \partial_{\perp}^{2} A_{-}+\frac{1}{C(R)} \int d^{4} x \mathcal{T}_{-} \partial_{\perp}^{2} A_{+} \\
& +\frac{1}{C(R)} \int d^{4} x J_{-} A \\
& \quad+\frac{1}{C(R)} \int d^{4} x J_{+} A_{-}-\frac{1}{2 C(R)} \\
& \left.\quad \times \int d^{4} x J_{+}\left(\partial_{\perp}^{2}\right)^{-1} J_{-}\right) .
\end{aligned}
$$

Finally, taking the external currents equal to zero, we write the generating functional for Lipatov's action in the Euclidean space:

$$
\begin{aligned}
Z\left[A_{+}, A_{-}\right]= & \frac{1}{Z^{\prime}} \int D v \exp \left(-S_{Y M}[v]-\frac{2}{C(R)}\right. \\
& \times \int d^{4} x A_{+}(x) \partial_{\perp}^{2} A_{-}(x) \\
& +\frac{1}{C(R)} \int d^{4} x \mathcal{T}_{+} \partial_{\perp}^{2} A_{-}+\frac{1}{C(R)} \\
& \left.\times \int d^{4} x \mathcal{T}_{-} \partial_{\perp}^{2} A_{+}\right) .
\end{aligned}
$$

The classical equations of motion are in the case

$$
\left(D_{\mu} G^{\mu \nu}\right)_{a}=\partial_{\mu} G_{a}^{\mu \nu}+g f_{a b c} v_{\mu}^{b} G^{c \mu \nu}=j_{a}^{v}
$$

with the new effective currents obtaining by the variation of the Lipatov's currents with respect to the $v_{4}, v_{1}$ gluon fields. The details of the derivation of the current can be found in [8], we have then:

$$
\begin{aligned}
j_{a}^{4}= & -\frac{1}{N \sqrt{2}} \operatorname{tr}\left[f_{a} O_{+} f_{b} O_{+}^{T}\right]\left(\partial_{\perp}^{2} A_{-}^{b}\right) \\
& -\frac{1}{N \sqrt{2}} \operatorname{tr}\left[f_{a} O_{-} f_{b} O_{-}^{T}\right]\left(\partial_{\perp}^{2} A_{+}^{b}\right) \\
j_{a}^{1}= & -\frac{1}{N \sqrt{2}} \operatorname{tr}\left[f_{a} O_{+} f_{b} O_{+}^{T}\right]\left(\partial_{\perp}^{2} A_{-}^{b}\right) \tan (\phi) \\
& +\frac{1}{N \sqrt{2}} \operatorname{tr}\left[f_{a} O_{-} f_{b} O_{-}^{T}\right]\left(\partial_{\perp}^{2} A_{+}^{b}\right) \tan (\phi)
\end{aligned}
$$

which to LO are equal to

$$
\begin{aligned}
& j_{a}^{4}=\frac{1}{\sqrt{2}} \partial_{\perp}^{2}\left(A_{-}+A_{+}\right), \\
& j_{a}^{1}=\frac{1}{\sqrt{2}} \partial_{\perp}^{2}\left(A_{-}-A_{+}\right) \tan (\phi) .
\end{aligned}
$$

\footnotetext{
${ }^{2}$ In Minkowski space these fields are Reggeon fields.
}

In the case of the fundamental representation we obtain instead Eq. (35):

$$
\begin{aligned}
j_{a}^{4}= & -\frac{l}{N \sqrt{2}} \operatorname{tr}\left[f_{a} O_{+} f_{b} O_{+}^{T}\right]\left(\partial_{\perp}^{2} A_{-}^{b}\right) \\
& -\frac{l}{N \sqrt{2}} \operatorname{tr}\left[f_{a} O_{-} f_{b} O_{-}^{T}\right]\left(\partial_{\perp}^{2} A_{+}^{b}\right) \\
j_{a}^{1}= & -\frac{l}{N \sqrt{2}} \operatorname{tr}\left[f_{a} O_{+} f_{b} O_{+}^{T}\right]\left(\partial_{\perp}^{2} A_{-}^{b}\right) \tan (\phi) \\
& +\frac{l}{N \sqrt{2}} \operatorname{tr}\left[f_{a} O_{-} f_{b} O_{-}^{T}\right]\left(\partial_{\perp}^{2} A_{+}^{b}\right) \tan (\phi) .
\end{aligned}
$$

In general we can redefine the Reggeon fields

$A_{+} \rightarrow \imath A_{+}, A_{-} \rightarrow \imath A_{-}$

obtaining in the case the same classical equation Eq. (35) and Eq. (37) in terms of redefined auxiliary fields.

\section{The action in Euclidean "light-cone" coordinates}

The presence of the angle in Eq. (35) currents determine the gluons fields as dependent on the angle through the equations of motion. Whereas these expressions are suitable for the analytical calculations, the numerical implementation of any calculations can be complicated somehow because of the angle present. Therefore, we introduce the following "light-cone" coordinates in the Euclidean space. Requiring $n_{ \pm}^{\mu} \partial_{\mu} O_{ \pm}=\partial_{ \pm}$we determine the "contravariant" "lightcone" coordinates as

$x^{+}=\frac{x^{4}+x^{1} / \tan \phi}{\sqrt{2}}, x^{-}=\frac{x^{4}-x^{1} / \tan \phi}{\sqrt{2}}$

and "covariant" gluon fields in the Euclidean space as

$v_{+}=\frac{v_{4}+v_{1} \tan \phi}{\sqrt{2}}, \quad v_{-}=\frac{v^{4}-v^{1} \tan \phi}{\sqrt{2}}$

Corresponding "covariant" and "contravariant" vectors are obtained with the help of the following metric tensor ${ }^{3}$ :

$$
\begin{aligned}
g_{\mu \nu} & =\frac{1}{2 \cos ^{2}(\phi)}\left(\begin{array}{cc}
1 & \cos (2 \phi) \\
\cos (2 \phi) & 1
\end{array}\right), \\
g^{\mu \nu} & =\frac{1}{2 \sin ^{2}(\phi)}\left(\begin{array}{cc}
1 & -\cos (2 \phi) \\
-\cos (2 \phi) & 1
\end{array}\right), \mu v=+-.
\end{aligned}
$$

\footnotetext{
3 The tensor convert form of Eq. (41) vectors to Eq. (42) form and vise verse, it's action is given simply by $x^{ \pm}(\phi)=x_{ \pm}(\pi / 2-\phi)$ replace.
} 
In this case we have for the Lagrangian:

$$
\begin{aligned}
L_{Q C D}= & \frac{1}{2} G_{+-}^{a} G_{a}^{+-}+\frac{1}{2} G_{+i}^{a} G_{a}^{+i} \\
& +\frac{1}{2} G_{-i}^{a} G_{a}^{-i}+\frac{1}{4} G_{i j}^{a} G_{a}^{i j}
\end{aligned}
$$

and for the effective currents Eq. (28):

$$
\begin{aligned}
& O_{+}=e^{g \int_{-\infty}^{x^{+}} d x^{\prime}+v_{+}\left(x^{\prime}+, x^{-}, x_{\perp}\right)}, \\
& O_{-}=e^{g \int_{-\infty}^{x^{-}} d x^{\prime}-v_{-}\left(x^{+}, x^{\prime}-, x_{\perp}\right)} .
\end{aligned}
$$

We see, therefore, that in these "light-cone" coordinates the Eq. (33) effective action does not contain the angle, it must be accounted only once in the Eq. (42) definition of "covariant" and corresponding "contravariant" gluon fields. The price for that is the doubled number of the longitudinal gluon fields which are depend each on other trough the transformations with the use of Eq. (43) metric tensor. Additional advantage of the introduced coordinates is that we can use here one from the $v_{ \pm}=0$ gauges that simplifies the structure of the Eq. (45) currents terms and corresponding numerical calculations.

\section{The action in axial gauge}

The "light-cone" coordinates introduced above are not so suitable for the analytical calculation and construction of the Euclidean Regge Field Theory (RFT). Eliminating the angle's dependence of the effective currents, the new gluons fields "move" the angle in the 1.h.s. of Eq. (34) written in the terms of only "covariant" coordinates. The Lagrangian Eq. (44) does not help in this case, there is no simple rule which allows to raise and lower the corresponding indexes with the help of Eq. (43) tensor. Therefore, we consider the analytical solution of Eq. (34) equations of motion taking $v_{1}=0$ axial gauge, where in this case the condition $O_{+}=O_{-}$also is satisfied. We have correspondingly to LO precision: ${ }^{4}$

$$
\begin{aligned}
& \partial_{4}^{2} v_{i}+\partial_{1}^{2} v_{i}-\partial_{4} \partial_{i} v_{4}=0 \\
& \partial_{1}^{2} v_{4}+\partial_{i}^{2} v_{4}-\partial_{4} \partial_{i} v_{i}=j_{4} \\
& -\partial_{4} \partial_{1} v_{4}-\partial_{i} \partial_{1} v_{i}=j_{1} .
\end{aligned}
$$

The solution of the equations are the following functions:

$$
\begin{aligned}
& v_{4}^{c l}\left(A_{+}, A_{-}\right)=\square^{-1}\left(j_{4}-\partial_{4} \partial_{1}^{-1} j_{1}\right), \\
& v_{i}^{c l}\left(A_{+}, A_{-}\right)=-\partial_{i} \square^{-1}\left(\partial_{1}^{-1} j_{1}\right) .
\end{aligned}
$$

\footnotetext{
${ }^{4}$ We omit here color indexes of the fields for the shortness of the notations.
}

The third equation from the system for the two unknown functions is the condition of transversality of the currents:

$\partial_{\mu} j_{\mu}=0$

that to LO can be written with the help of Eq. (37) as:

$$
\begin{aligned}
\partial_{+} A_{-} & =\partial_{-} A_{+}=0 \rightarrow A_{-} \\
& =A_{-}\left(x^{-}\right), A_{+}=A_{+}\left(x^{+}\right),
\end{aligned}
$$

see definitions in the above section.

\section{Instantons in the effective action framework}

Now we ready to shortly sketch out the possibility of the accounting of the classical instanton solution in the framework of the effective action. There are plenty of the different applications of the instanton contributions to the high energy scattering processes, see [69-86] and references therein. The effective action, in turn, provide a framework where these instanton's contributions can be, in principle, accounted on the level of the systematic perturbative calculations. Indeed, the gauge introduced in the above section is useful for the definition of the instantons in YM theory, see [95-100], and can be used effectively for the description of the Reggeon solution. The general idea of the calculation which we propose is the following therefore. The instanton is a classical non-perturbative solution of the homogenous Euclidean YM equations of motion, see [95-100] for example. Then, the gluon fields in Euclidean space we can write as as classical solution of homogenous equation plus classical solution of non-homogenous equation (the Reggeon solution) plus fluctuations around it:

$$
\begin{aligned}
& v_{4}=v_{4}^{c l}+\varepsilon_{4}=v_{4}^{i n s t}+v_{4}^{c l}\left(A_{+}, A_{-}, v^{i n s t}\right)+\varepsilon_{4}, \\
& v_{i}=v_{i}^{c l}+\varepsilon_{i}=v_{i}^{i n s t}+v_{i}^{c l}\left(A_{+}, A_{-}, v^{i n s t}\right)+\varepsilon_{i} .
\end{aligned}
$$

Here the dependence of the $v_{4 i}^{c l}$ classical Reggeon solution on the instanton fields will arise from the NLO of the perturbation theory due the non-linearity of the equations of motion, whereas to the LO precision only the whole classical solution is a linear combination of the instanton and Reggeon fields. Inserting the Eq. (52) expressions back into the action, we obtain for Eq. (33) generating functional:

$$
\begin{aligned}
Z\left[v^{i n s t}, A_{+}, A_{-}\right]= & \frac{1}{Z^{\prime}} \int D \varepsilon \exp \left(-S_{Y M}[v]-\frac{2}{C(R)}\right. \\
& \times \int d^{4} x A_{+}(x) \partial_{\perp}^{2} A_{-}(x) \\
& +\frac{1}{C(R)} \int d^{4} x \mathcal{T}_{+}(v) \partial_{\perp}^{2} A_{-}+\frac{1}{C(R)} \\
& \left.\times \int d^{4} x \mathcal{T}_{-}(v) \partial_{\perp}^{2} A_{+}\right) .
\end{aligned}
$$


This functional effectively determines the vertices of interactions of $A_{ \pm}$fields with the instanton fields in the framework of high energy Euclidean QCD RFT, after the inverse continuation to Minkowski space it will determine the vertices of interactions of Reggeon with instanton fields as well.

We note, that using the diagrammatic approach of [37,14-27], the effective currents determine the Feynman rules for the construction of the vertices of interaction of gluons with instanton and Reggeon fields. Namely, instead the Eq. (52) representation of gluon fields, the any interaction vertex of interest can be constructed by the $v \rightarrow v+v^{i n s t}$ substitution performed directly in the effective currents and their consequent expansion into the perturbative series similarly to done in [3-7].

\section{Conclusion}

The obtained Eqs. (33), (45) and Eqs. (50)-Eq. (53) expressions we consider as the main results of the paper which clarify two issues concerning the formalism of high energy QCD Lipatov's effective action in Euclidean space. Namely, the formalism is expanded to Euclidean space on purpose of the following possible applications of the Euclidean version of the action.

The first one is a possible implementation of the numerical (lattice) calculation in the framework with the Euclidean action. With the help of Eq. (33) generating functional and Eq. (45) effective currents expressions, any correlator of $A_{ \pm}$ fields can be calculated. ${ }^{5}$ In Minkowski space it will allow to trace the high energy behavior of the arbitrary Reggeon's correlators. For example, taking BFKL colorless state we can calculate the correlator in Euclidean state for the different values of $\phi$ angle. The reverse continuation to Minkowski space, therefore, will allow to interpolate the behavior of the correlator as function of energy on the base of the points obtained in the Euclidean space. These non-perturbative calculations of the high energy asymptotic behavior of the Pomeron (and other correlators) with the unitarity corrections included is an interesting task due the importance of the BFKL calculus in the high-energy QCD. Another interesting possibility of the application of the formalism is the connection of the Wilson lines correlators and correlators of $A_{ \pm}$fields, see Eqs. (31)(32), the knowledge of $A_{ \pm}$correlators will determine the correlators of Wilson lines as well. It is important to underline, nevertheless, that the obtained action is the only first step towards to the real lattice calculations which definitely will require a lot of additional work to do. For example, the discretization of the effective currents, introducing Wilson

\footnotetext{
5 For the color correlators an additional regularization of the effective currents must be introduced.
}

loops and etc. must be done in the framework as well, these tasks we will consider in the separate publications.

Another application of the Euclidean version of the action is that it demonstrates a possibility of the definition of the correct interaction vertices of the correlators of $A_{ \pm}$fields with the instantons. Namely, Eq. (53), after the integration with respect to the classical instanton fields, will provide instanton induced corrections to the Reggeon fields correlators, i.e. to the propagator of reggeized gluons, BFKL Pomeron, et cetera. These corrections are interesting to account, see [69-86] for the different applications of the instanton contributions in the high energy scattering processes. An opposite is also true, it is a possibility to define a contribution of the Reggeon fields into the instanton solutions by initial integration of the Reggeon fields in the generating functional. The calculations related to that problem is an especially interesting task, we consider it as the next step in the development of the framework.

Data Availability Statement This manuscript has no associated data or the data will not be deposited. [Authors' comment: This is a theoretical study and no experimental data has been listed.]

Open Access This article is licensed under a Creative Commons Attribution 4.0 International License, which permits use, sharing, adaptation, distribution and reproduction in any medium or format, as long as you give appropriate credit to the original author(s) and the source, provide a link to the Creative Commons licence, and indicate if changes were made. The images or other third party material in this article are included in the article's Creative Commons licence, unless indicated otherwise in a credit line to the material. If material is not included in the article's Creative Commons licence and your intended use is not permitted by statutory regulation or exceeds the permitted use, you will need to obtain permission directly from the copyright holder. To view a copy of this licence, visit http://creativecomm ons.org/licenses/by/4.0/.

Funded by $\mathrm{SCOAP}^{3}$.

\section{References}

1. L.N. Lipatov, Nucl. Phys. B 452, 369 (1995)

2. L.N. Lipatov, Phys. Rept. 286, 131 (1997)

3. E.N. Antonov, L.N. Lipatov, E.A. Kuraev, I.O. Cherednikov, Nucl. Phys. B 721, 111 (2005)

4. L.N. Lipatov, Subnucl. Ser. 49, 131 (2013)

5. L.N. Lipatov, Int. J. Mod. Phys. Conf. Ser. 39, 1560082 (2015)

6. L.N. Lipatov, Int. J. Mod. Phys. A 31(28/29), 1645011 (2016)

7. L.N. Lipatov, EPJ Web Conf. 125, 01010 (2016)

8. S. Bondarenko, L. Lipatov, A. Prygarin, Eur. Phys. J. C 77(8), 527 (2017)

9. S. Bondarenko, L. Lipatov, S. Pozdnyakov, A. Prygarin, Eur. Phys. J. C 77(9), 630 (2017)

10. S. Bondarenko, S.S. Pozdnyakov, Phys. Lett. B 783, 207 (2018)

11. S. Bondarenko, M.A. Zubkov, Eur. Phys. J. C 78(8), 617 (2018)

12. S. Bondarenko, S.S. Pozdnyakov, Int. J. Mod. Phys. A 33(35), 1850204 (2018)

13. S. Bondarenko, S. Pozdnyakov, arXiv:1903.11288 [hep-th]

14. L.N. Lipatov, Nucl. Phys. Proc. Suppl. 99A, 175 (2001)

15. M.A. Braun, M.I. Vyazovsky, Eur. Phys. J. C 51, 103 (2007) 
16. M.A. Braun, M.Y. Salykin, M.I. Vyazovsky, Eur. Phys. J. C 65 , 385 (2010)

17. M.A. Braun, L.N. Lipatov, M.Y. Salykin, M.I. Vyazovsky, Eur. Phys. J. C 71, 1639 (2011)

18. M.A. Braun, M.Y. Salykin, M.I. Vyazovsky, Eur. Phys. J. C 72, 1864 (2012)

19. M. Hentschinski, A. Sabio Vera, Phys. Rev. D 85, 056006 (2012)

20. M.A. Braun, M.Y. Salykin, S.S. Pozdnyakov, M.I. Vyazovsky, Eur. Phys. J. C 72, 2223 (2012)

21. J. Bartels, L.N. Lipatov, G.P. Vacca, Phys. Rev. D 86, 105045 (2012)

22. M.A. Braun, S.S. Pozdnyakov, M.Y. Salykin, M.I. Vyazovsky, Eur. Phys. J. C 73(9), 2572 (2013)

23. G. Chachamis, M. Hentschinski, J.D. Madrigal Martínez, A. Sabio Vera, Phys. Part. Nucl. 45(4), 788 (2014)

24. M.A. Braun, Eur. Phys. J. C 75(7), 298 (2015)

25. M.A. Braun, M.I. Vyazovsky, Phys. Rev. D 93(6), 065026 (2016)

26. M.A. Braun, Eur. Phys. J. C 77(5), 279 (2017)

27. M.A. Braun, M.Y. Salykin, Eur. Phys. J. C 77(7), 498 (2017)

28. L.N. Lipatov, M.I. Vyazovsky, Nucl. Phys. B 597, 399 (2001)

29. M.A. Nefedov, V.A. Saleev, A.V. Shipilova, Phys. Rev. D 87(9), 094030 (2013)

30. A.V. Karpishkov, M.A. Nefedov, V.A. Saleev, A.V. Shipilova, Phys. Rev. D 91(5), 054009 (2015)

31. M. Nefedov, V. Saleev, Phys. Rev. D 92(9), 094033 (2015)

32. M. Nefedov, V. Saleev, On the one-loop calculations with Reggeized quarks. Mod. Phys. Lett. A 32(40), 1750207 (2017)

33. A.V. Karpishkov, M.A. Nefedov, V.A. Saleev, A.V. Shipilova, Phys. Part. Nucl. 48(5), 827 (2017). [Fiz. Elem. Chast. Atom. Yadra 48 (2017) no.5 ]

34. A.V. Karpishkov, M.A. Nefedov, V.A. Saleev, Phys. Rev. D 96(9), 096019 (2017)

35. M.A. Nefedov, arXiv:1902.11030 [hep-ph]

36. V.N. Gribov, Sov. Phys. JETP 26, 414 (1968)

37. D. Amati, L. Caneschi, R. Jengo, Nucl. Phys. B 101, 397 (1975)

38. R. Jengo, Nucl. Phys. B 108, 447 (1976)

39. D. Amati, M. Le Bellac, G. Marchesini, M. Ciafaloni, Nucl. Phys. B 112, 107 (1976)

40. M. Ciafaloni, M. Le Bellac, G.C. Rossi, Nucl. Phys. B 130, 388 (1977)

41. M. Ciafaloni, Nucl. Phys. B 146, 427 (1978)

42. A.H. Mueller, Nucl. Phys. B 437, 107 (1995)

43. P. Rembiesa, A.M. Stasto, Nucl. Phys. B 725, 251 (2005)

44. A.I. Shoshi, B.W. Xiao, Phys. Rev. D 73, 094014 (2006)

45. M. Kozlov, E. Levin, Nucl. Phys. A 779, 142 (2006)

46. A.I. Shoshi, B.W. Xiao, Phys. Rev. D 73, 094014 (2006)

47. M.A. Braun, G.P. Vacca, Eur. Phys. J. C 50, 857 (2007)

48. J.P. Blaizot, E. Iancu, D.N. Triantafyllopoulos, Nucl. Phys. A 784, 227 (2007)

49. S. Bondarenko, L. Motyka, A.H. Mueller, A.I. Shoshi, B.-W. Xiao, Eur. Phys. J. C 50, 593 (2007)

50. N. Armesto, S. Bondarenko, J.G. Milhano, P. Quiroga, JHEP 0805, 103 (2008)

51. S. Bondarenko, Eur. Phys. J. C 71, 1587 (2011)

52. S. Bondarenko, L. Horwitz, J. Levitan, A. Yahalom, Nucl. Phys. A 912, 49 (2013)

53. S. Bondarenko, L. Motyka, Phys. Rev. D 75, 114015 (2007)

54. S. Bondarenko, M.A. Braun, Nucl. Phys. A 799, 151 (2008)

55. S. Bondarenko, E. Gotsman, E. Levin, U. Maor, Nucl. Phys. A 683, 649 (2001)
56. S. Ostapchenko, Phys. Lett. B 636, 40 (2006)

57. S. Ostapchenko, Phys. Rev. D 77, 034009 (2008)

58. S. Ostapchenko, Phys. Rev. D 81, 114028 (2010)

59. E. Levin, J. Miller, A. Prygarin, Nucl. Phys. A 806, 245 (2008)

60. E. Levin, A. Prygarin, Eur. Phys. J. C 53, 385 (2008)

61. M.A. Braun, A.N. Tarasov, Eur. Phys. J. C 58, 383 (2008)

62. M.A. Braun, Eur. Phys. J. C 63, 287 (2009)

63. T. Altinoluk, A. Kovner, M. Lublinsky, J. Peressutti, JHEP 0903, 109 (2009)

64. M.A. Braun, A. Tarasov, Eur. Phys. J. C 69, 75 (2010)

65. R.S. Kolevatov, K.G. Boreskov, L.V. Bravina, Eur. Phys. J. C 71, 1757 (2011)

66. M.A. Braun, A.N. Tarasov, Phys. Lett. B 726, 300 (2013)

67. T. Altinoluk, A. Kovner, E. Levin, M. Lublinsky, JHEP 1404, 075 (2014)

68. A.E. Bolshov, L.V. Bork, A.I. Onishchenko, JHEP 1806, 129 (2018)

69. S.Y. Khlebnikov, V.A. Rubakov, P.G. Tinyakov, Nucl. Phys. B 350, 441 (1991)

70. A.H. Mueller, Nucl. Phys. B 348, 310 (1991)

71. A.H. Mueller, Nucl. Phys. B 364, 109 (1991)

72. V.A. Rubakov, P.G. Tinyakov, Phys. Lett. B 279, 165 (1992)

73. V.I. Zakharov, Nucl. Phys. B 371, 637 (1992)

74. P.G. Tinyakov, Int. J. Mod. Phys. A 8, 1823 (1993)

75. I.I. Balitsky, V.M. Braun, Phys. Rev. D 47, 1879 (1993)

76. I.I. Balitsky, V.M. Braun, Phys. Lett. B 314, 237 (1993)

77. I.I. Balitsky, V.M. Braun, Phys. Lett. B 346, 143 (1995)

78. E.V. Shuryak, I. Zahed, Phys. Rev. D 62, 085014 (2000)

79. M.A. Nowak, E.V. Shuryak, I. Zahed, Phys. Rev. D 64, 034008 (2001)

80. D.E. Kharzeev, Y.V. Kovchegov, E. Levin, Nucl. Phys. A 690, 621 (2001)

81. A.E. Dorokhov, I.O. Cherednikov, Phys. Rev. D 66, 074009 (2002)

82. F. Schrempp, A. Utermann, Phys. Lett. B 543, 197 (2002)

83. G.W. Carter, D.M. Ostrovsky, E.V. Shuryak, Phys. Rev. D 65, 074034 (2002)

84. D.E. Kharzeev, Y.V. Kovchegov, E. Levin, Nucl. Phys. A 699, 745 (2002)

85. A.E. Dorokhov, I.O. Cherednikov, Ann. Phys. 314, 321 (2004)

86. A.E. Dorokhov, I.O. Cherednikov, Fiz. Elem. Chast. Atom. Yadra 35, 597 (2004)

87. I. Balitsky, Nucl. Phys. B 463, 99 (1996)

88. G. Chachamis, M. Hentschinski, J. D. Madrigal Martinez, A. Sabio Vera, Nucl. Phys. B 876, 453-472 (2013)

89. M. Hentschinski, A. Sabio Vera, Phys. Rev. D85, 056006 (2012)

90. G. Chachamis, M. Hentschinski, J. D. Madrigal Martinez, A. Sabio Vera, Phys. Rev. D87, 076009 (2013)

91. M. Nefedov, V. Saleev, Mod. Phys. Lett. A 32, 1750207 (2017)

92. M. A. Nefedov, arXiv:1902.11030 (2019)

93. E. Meggiolaro, Z. Phys. C 76, 523 (1997)

94. M. Giordano, E. Meggiolaro, Phys. Lett. B 675, 123 (2009)

95. D.J. Gross, R.D. Pisarski, L.G. Yaffe, Rev. Mod. Phys. 53, 43 (1981)

96. A.I. Vainshtein, V.I. Zakharov, V.A. Novikov, M.A. Shifman, Sov. Phys. Usp. 25, 195 (1982)

97. D. Diakonov, Prog. Part. Nucl. Phys. 51, 173 (2003)

98. A. Smilga, Lectures on Quantum Chromodynamics (World Scientific, Singapore, 2001)

99. J. Zinn-Justin, U.D. Jentschura, Ann. Phys. 313, 197 (2004)

100. M. Mariño, Fortsch. Phys. 62, 455 (2014) 